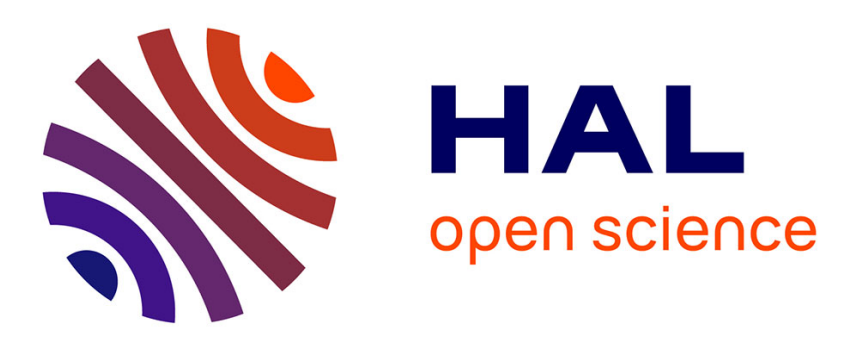

\title{
Méditerranée, Moyen-Orient : Les trois états d'une génération
}

Pierre Blanc

\section{To cite this version:}

Pierre Blanc. Méditerranée, Moyen-Orient : Les trois états d'une génération. Confluences Méditerranée , 2017, 100, pp.13-25. 10.3917/come.100.0013 . halshs-02189440

\section{HAL Id: halshs-02189440 \\ https://shs.hal.science/halshs-02189440}

Submitted on 22 Jul 2019

HAL is a multi-disciplinary open access archive for the deposit and dissemination of scientific research documents, whether they are published or not. The documents may come from teaching and research institutions in France or abroad, or from public or private research centers.
L'archive ouverte pluridisciplinaire HAL, est destinée au dépôt et à la diffusion de documents scientifiques de niveau recherche, publiés ou non, émanant des établissements d'enseignement et de recherche français ou étrangers, des laboratoires publics ou privés. 


\section{Méditerranée, Moyen-Orient : les trois états d'une génération}

\section{Pierre Blanc}

Les Afriques dans le monde, CNRS/Sciences po Bordeaux. Rédacteur en chef de Confluences Méditerranée.

25 ans. Une génération. C'est aussi l'âge de Confluences Méditerranée. Nous sommes heureux de cette aventure partagée, stimulante et durable. En 25 ans, la revue n'a jamais manqué une sortie trimestrielle. Avec le présent numéro, nous en sommes donc rendus au centième. Le premier fut publié à la fin 1991, c'est-à-dire à un tournant historique pour le monde et la région : celui de la fin de la guerre froide et du début des négociations de Madrid entre Israéliens et Arabes qui pouvaient permettre d'envisager un avenir meilleur. 25 ans après, nous avons voulu consacrer le numéro 100 au bilan de cette période. Si au début de cette ère nombre de commentateurs et politologues purent imaginer l'avenir en des termes favorables, et si beaucoup de citoyens pouvaient se réjouir d'entrevoir la guérison des fractures du monde, l'espoir né de quelques promesses semble avoir malheureusement laissé la place à l'amertume du constat. En particulier en Méditerranée et au Moyen-Orient où la violence politique, loin d'avoir disparu, a gagné en intensité tandis que les arènes du conflit n'ont jamais été aussi nombreuses.

\section{Promesses}

Le début de la décennie 1990 a constitué une rupture historique. Après avoir structuré les relations internationales pendant cinq décennies, le paradigme de la guerre froide a sombré avec la dislocation de l'URSS. Entérinée en 1991, c'est en fait dès 1989 que l'URSS a démontré au monde entier qu'elle s'était départie de sa prétention impériale. Les révolutions de velours dans les pays de l'Europe de l'Est étaient la meilleure traduction de cette nouvelle politique voulue par Mikhail Gorbatchev. Avec la publication d'un article la même année dans The National Interest, Francis Fukuyama pouvait ainsi se faire le héraut de «la fin de l'histoire », comme Karl Marx l'avait fait en son temps. Mais si pour celui-ci la révolution prolétarienne devait arrêter le moteur historique de la lutte des classes, Fukuyama voyait dans la généralisation de la démocratie libérale la raison de la sortie de l'histoire. Plus ou moins rapidement, tous les espaces se convertiraient au parlementarisme et à l'économie de marché. Et, en écho aux travaux de Michael Doyle qui considère que les démocraties ne se font pas la guerre, Fukuyama entrevoyait dans cette conversion le moyen le plus puissant de stériliser les terreaux de la guerre, 
même s'il reconnaissait que des zones allaient demeurer pour un temps dans l'histoire. Ce raisonnement aux accents prophétiques lui valut beaucoup de critiques, et notamment celles de Samuel Huntington qui répliqua avec un article «No exit, The errors of endism »(Pas de sortie, la fin du finisme). En dépit de ce feu de critiques, Fukuyama développa sa théorie dans son fameux ouvrage sur la fin de l'histoire publié en 1992.

Ce qui se passait alors au Moyen-Orient n'était sans doute pas fait pour le détourner de sa thèse. Cette région, véritable épicentre de l'instabilité internationale, pouvait entrer dans son récit « eschatologique ». Pourtant, l'Irak avait envahi le Koweït dans la nuit du 2 août 1990 mais le contexte de sortie de l'affrontement entre l'Est et l'Ouest avait sans doute facilité le vote d'une résolution onusienne visant à contraindre l'Irak de sortir du territoire agressé. La communauté internationale semblait donc ré-exister avec l'adoption par le Conseil de sécurité de la résolution 678 (sous chapitre 7), qui autorisa alors «l'emploi de tous les moyens » pour revenir au statu quo ante. En janvier 1991, le président américain avait pu déclarer de façon enthousiaste devant le congrès: "Nous avons devant nous l'opportunité de forger pour nous-mêmes et les générations à venir un nouvel ordre dans le monde, un ordre où le droit et pas la loi de la jungle, dirigera le comportement des nations. »

La question du Koweït fut finalement réglée par le départ forcé des troupes irakiennes en février 1991. Ce succès apparent fut suivi par le massacre des Kurdes et des Chiites d'Irak. Après avoir été encouragés par les Américains à se révolter contre Saddam Hussein, ils eurent à subir ses foudres les plus brutales. Plus largement, les Irakiens furent écrasés par un embargo meurtrier décidé à la suite de cette guerre éclair.

Nonobstant ces fautes tragiques, le nouvel ordre international, qui participait davantage de la pax americana que d'un véritable multilatéralisme, sembla trouver un nouveau terrain d'investissement avec le conflit israélo-arabe si central dans l'instabilité internationale. Pour faire avancer cette idée, et alors qu' Israël, dirigé par le Likoud de Shamir, y était frontalement hostile, Washington fit pression pour la première fois sur son allié. Le refus d'une garantie bancaire destinée à obtenir un prêt de 10 milliards de dollars, tant que se développait la colonisation dans les territoires palestiniens, sembla porter ses fruits. Les travaillistes revenus au pouvoir en juin 1992 prirent en effet acte de cette menace et s'engagèrent dans ce dialogue israélo-arabe puis dans le processus de paix israélo-palestinien à partir de 1993. Même s'ils ne manquaient pas d'opposants (notamment les partisans du Grand Isräl d'un côté et le Hamas de l'autre) et de sceptiques (qui avaient quelques raisons de l'être), les accords d'Oslo se présentaient comme un jalon encourageant sur le chemin de la paix entre Palestiniens et 
Israéliens mais également entre ceux-ci et les Arabes car la centralité du conflit de Palestine est au cœur de leur différend.

Le nouvel ordre international semblait donc poursuivre son œuvre, d'autant plus que les transitions démocratiques entamées durant la décennie 1980 en Amérique du Sud et le lancement de conférences nationales en Afrique en ce début de décennie 1990, validaient en apparence cette perspective. Sur ce continent, l'effondrement du régime d'apartheid en Afrique du Sud, figure même de l'abomination politique, avait fourni sans doute la nouvelle la plus encourageante en ce sens. L'attribution du prix Nobel de la Paix au président Frederik de Klerk et à Nelson Mandela en était le signe. L'année suivante, l'attribution du même prix à Yasser Arafat et Yitzhak Rabin situait aussi l'importance du chemin parcouru au Moyen-Orient.

Dans cette dynamique et pour peser sur l'histoire, la rive nord de la Méditerranée s'engagea davantage sur les rives est et sud, appelant à la construction progressive d'une zone de coprospérité. Le partenariat euro-méditerranéen, engagé à Barcelone en 1995, se voulait porteur d'une dynamique culturelle et économique commune au service d'un apaisement politique régional. «Un espace de paix, de sécurité et de prospérité partagée » telle était l'antienne !

L'Europe avait besoin de paix. Par son histoire, elle savait le prix de la guerre. Par son voisinage, elle en connaissait son actualité. 1995 sonnait en effet la fin de la tragédie yougoslave (à l'exception de la question du Kosovo) où la braise des nationalismes avait fini par enflammer le toit politique de cette fédération des slaves du sud. Ces peuples de la bordure méditerranéenne, qui avaient voulu partager un destin commun en 1919 puis en 1945, ne le désiraient plus en ce début de décennie 1990. Dans la foulée de l'effondrement de l'URSS et de ses satellites, l'organisation d'élections dans les républiques fédérées avait donné la victoire à des nationalistes qui étaient dans bien des cas d'anciens dévots du communisme. Reconvertis idéologiquement, ils purent s'exercer en entrepreneurs de violence pour purifier leurs territoires et commettre de nombreux crimes contre l'humanité. Cet espace connut encore un dernier épisode très violent entre 1998 et 1999 avec la guerre au Kosovo entre nationalistes serbes et albanais. A la fin de ce conflit, l'établissement de la KFOR (Kosovo force) sous mandat onusien, apparaissait comme une lumière bien pâle de ce nouvel ordre international que George Bush avait entrevu si flamboyant.

\section{Désenchantements}

Son successeur, Bill Clinton, qui avait parrainé en 1993 l'entrée dans le processus de paix entre Israéliens et Palestiniens, pouvait faire le constat à ce tournant des années 1990 que la poignée 
de main entre Arafat et feu Rabin, assassiné en 1994 par Yigal Amir, un Israélien radicalisé, n'avait finalement rien donné de concret sur le terrain. Pire : Israël, servi (ou aveuglé) par un rapport de force profondément asymétrique avec les Palestiniens ${ }^{1}$ avait continué à déployer une stratégie d'emprise territoriale en Cisjordanie malgré les engagements pris par les signataires d'Oslo. En 2000, soit cinq ans après Oslo II, le constat s'imposait d'une colonisation toujours plus poussée et d'un grignotage territorial de la Cisjordanie dont la plus grande partie (les fameuses zones C) était laissée sous le contrôle absolu d'Israël. Quant à Gaza, la vie y était de plus en plus impossible eu égard à son enfermement croissant.

Oublieux de son parrainage pendant ses deux mandats, Bill Clinton tenta à la fin de son deuxième de relancer un processus au point mort, en réunissant les leaders palestiniens et israéliens à Camp David au cours de l'été 2000. Les propositions ne furent finalement pas acceptées par les Palestiniens car selon le conseiller du président américain, Robert Malley, qui reviendra sur cet échec et sur la duplicité de son administration, elles n'étaient tout simplement pas acceptables par eux, notamment sur les aspects territoriaux où les propositions de Camp David étaient très en deçà de l'esprit de résolutions 242 et 338 de l'ONU. Si du côté israélien, le discours officiel sur l'incapacité des Palestiniens à négocier déstabilisa complètement le camp de la paix, du côté palestinien, la frustration était à son comble. Faut-il s'étonner dès lors que la visite d'Ariel Sharon sur l'esplanade des mosquées le 28 septembre 2000 pour mieux dire que Jérusalem était une et indivisible provoqua le début de l'intifada Al-Aqsa ? Cet acte lui permit un regain de popularité dans une société de plus en plus droitisée qui l'amena au pouvoir en février 2001. Il profita de ce soulèvement palestinien, il est vrai armé contrairement à la première intifada de 1987, pour détruire les attributs de l'Autorité palestinienne et plus largement le processus de paix.

Dans cette entreprise de démolition, Ariel Sharon fut clairement soutenu par George W. Bush, parvenu au pouvoir aux Etats-Unis en janvier 2001. Pour le nouveau président et ses thuriféraires néoconservateurs, le nouvel ordre international, basé sur l'exportation de la démocratie libérale, devait se construire aussi par le recours à la guerre, chaque fois qu'un Etat y répugnait... Les Etats voyous (wiw ? States), déjà nommés ainsi par Bill Clinton, étaient prioritaires sur la liste des néoconservateurs, maintenant au pouvoir, car ils formaient «l'axe du mal » selon la terminologie de la nouvelle administration. C'était le temps de la revanche aux Etats-Unis. Revanche contre le djihadisme qui avait fait

${ }^{1}$ Jean-Paul Chagnollaud, La défaite du vainqueur, Actes Sud, 2017. 
s'effondrer les Twin Towers à New-York le 11 septembre 2001. Francis Fukuyama persistait à ce moment-là ${ }^{2}$, voyant dans la chute meurtrière des tours jumelles un dernier avatar d'une logique parvenue à bout de souffle. De son côté, Samuel Huntington faisait de cet événement la validation $\mathrm{du}$ «choc de civilisation» dont il s'était fait le prophète quelques années auparavant. Sensible à ces deux théories, en dépit des divergences radicales qu'exprimaient leurs auteurs, l'administration américaine mit en scène un «militarisme théâtral » ${ }^{3}$ en Afghanistan et en Irak. Il s'agissait d'y exporter la démocratie tout en y détruisant les foyers djihadistes de la guerre de civilisation, qui n'existait pourtant pas chez le second.

En Irak, le régime de Saddam Hussein, d'une brutalité extrême, tomba rapidement après le début de l'invasion américano-britannique déclenchée au motif qu'il se faisait le complice d'AlQaida et qu'il détenait des armes de destructions massives. Du fait de sa brutalité, la fin du régime apparaissait loin d'être regrettable par-delà ces arguments fallacieux qu'avaient utilisés les Etats-Unis pour la faire advenir. Plus fâcheuse était la violation du droit international par Washington qui avait engagé la guerre sans l'aval de l'ONU. Pire encore allait être le bilan humain au fur et à mesure que la guerre civile s'installa en Irak. En procédant à une « débaasification » du pays, le directeur de la reconstruction et de l'assistance humanitaire, Paul Bremer, contribua aussi à marginaliser les sunnites qui, après avoir dominé le pays notamment en investissant les institutions du pourvoir baasiste, se trouvaient victimes d'une communauté chiite revancharde. En dépit d'une constitution fédérale décidée en 2005 qui permit de garder l'illusion d'un Etat « partagé », c'est bien à une revanche chiite, aussi inique que la suprématie des sunnites le fut (du moins celle du clan de Saddam), que l'on assista. Derrière les oripeaux de la revanche chiite, c'est surtout une nouvelle clique politique qui reproduisit le comportement prédateur de l'ancien régime. Cette conduite de la nouvelle oligarchie chiite, sur fond d'occupation américaine, fournissait un terreau au djihad sunnite qui allait ainsi trouver un nouveau souffle et dont les différents avatars allaient déboucher sur la création de Daech en juin 2014.

Trois ans après le début des révoltes arabes, la montée en puissance de cette secte néo-salafiste messianique traduisait le recul d'un mouvement parti de Tunisie en décembre 2010. Pourtant, tour à tour, des despotes étaient tombés (Ben Ali, Moubarak, Kadhafi et Ali Abdallah Saleh) tandis que Bachar el Assad avait semblé voué à subir le même sort. Ailleurs, les révoltes étaient beaucoup plus contenues, des changements de gouvernements et/ou quelques réformes

\footnotetext{
${ }^{2}$ «Nous sommes toujours à la fin de l'histoire », Le Monde, 18 octobre 2001.

${ }^{3}$ Emmanuel Todd, Après l'empire, Essai sur la décomposition du système américain, Gallimard, 2002.
} 
politiques ayant permis de prévenir une réelle révolution. Ainsi, pour reprendre les catégories d'analyse de Max Weber, il semble que les régimes forts à légitimité charismatique (Tunisie, Egypte, Libye, Syrie, Yémen), fondée sur l'émergence d'un homme puis d'un système, étaient bien parvenus à une fin de cycle ; a contrario, les régimes à légitimité traditionnelle, adossée à un arrière-plan dynastique et religieux (Jordanie, Arabie saoudite, Qatar, Maroc), restaient à l'écart du mouvement. La sollicitation de cette grille est à prendre avec beaucoup de précaution car d'autres éléments interviennent, notamment l'usage de la rente pétrolière : les Etats rentiers du Golfe, à légitimité traditionnelle, ont pu compter sur la richesse de leur sous-sol pour acheter la paix sociale.

Quant à l'Algérie républicaine, si la rente lui a été d'un précieux secours, c'est aussi le souvenir de la guerre civile des années 1990 qui a réfréné jusqu'à aujourd'hui les ardeurs révolutionnaires d'un peuple pauvre dans un pays riche.

Il n'empêche : en regardant les choses, certes grossièrement, les pouvoirs les plus menacés dans cette phase révolutionnaire furent bien les «républiques» arabes originellement charismatiques, dont le cycle historique a bien commencé dans les années 1950 et 1960 . Ce cycle avait été ouvert par des épisodes révolutionnaires et les nouveaux pouvoirs s'étaient engagés dans un «pacte social populiste ${ }^{4}$ où la souveraineté collective était prioritaire sur l'émancipation individuelle. En privilégiant l'émancipation de la nation sur celle de la personne, ces pouvoirs étaient d'emblée frappés d'un certain autoritarisme. Cependant, dans ce pacte tacite, l'économie devait amener aux populations un certain bien être social en échange de la confiscation de certaines libertés. Plusieurs décennies après ce premier virage révolutionnaire, le pacte ne fonctionnait plus : non seulement les sociétés étaient extrêmement cadenassées mais le chômage et les inégalités les minaient. Le manque d'emplois était devenu criant avec l'arrivée de classes d'âge jeune nombreuses sur un marché du travail atone. La stagnation de l'industrialisation - les pays arabes ont les taux d'emplois manufacturiers les plus faibles au monde - mais également les politiques d'ajustements structurels et de libéralisation conduites depuis les années 1980 jusqu'aux années 2000 avaient contribué à limiter les emplois. Ce mouvement de libéralisation avait en effet surtout permis à une oligarchie politique de capter l'outil de production sur fond de passe-droits exorbitants. Parmi les cas de néopatrimonialisme, faut- il rappeler le cas d'Ahmet Ezz, haut responsable du PND et magnat de l'acier, qui contrôlait tout le marché égyptien ? Faut-il évoquer l'exemple de Rami Makhlouf, le cousin de

${ }^{4} \mathrm{Je}$ reprends ici la formule de Laurence Louër. 
Bachar el Assad qui détenait nombre de licences d'importation et d'entreprises-clé dans son pays ? Faut-il également reparler des familles Ben ali Trabelsi qui, par le racket des entreprises tunisiennes, ont poussé celles-ci à autolimiter leurs investissements réduisant d'autant les emplois ? Tout ceci soulignait la profondeur de la corruption dans les sociétés arabes que le PNUD avait pointée en 2004.

Cet échec économique (chômage et inégalités) et moral (corruption) était d'autant plus insupportable que la privation des libertés était devenue intolérable et que les régimes s'étaient caricaturés dans leur brutalité : la peur était pensée comme clef du système d'emprise politique, de même que l'atomisation de la société avec les lois d'urgence de toutes sortes. En réalité cet écrasement et la longévité des régimes cachaient mal leur grande fragilité eu égard à leur faible légitimité auprès des sociétés qui avaient évolué en profondeur : plus formés, plus urbanisés, plus connectés à la mondialisation, certains pans de la société ne pouvaient plus tolérer ces régimes archaïques, brutaux et injustes. Cependant, il serait erroné d'analyser ces révoltes uniquement comme le soulèvement d'une avant-garde de la société civile car ces secteurs «avancés » furent rejoints par d'autres secteurs plus défavorisés de la société, à moins que ce ne fût le contraire comme en Tunisie où Sid Bouzid avait aiguillonné Tunis.

Six ans après le lancement de ce mouvement révolutionnaire, seule la Tunisie semble faire rimer transition politique et transition démocratique. Ailleurs, c'est le retour à l'autoritarisme (Egypte) et les guerres civiles (Syrie, Libye et Yémen) qui s'ajoutent à celle en Irak démarrée durant la décennie 2000. Ces Etats confisqués par des prédateurs politiques sont devenus des Etats faillis dont la pérennité se pose.

S'offrant comme un espoir, ce moment révolutionnaire est devenu un calvaire entre autres du fait des immixtions étrangères. En Libye, les Français, les Britanniques et les Américains, en outrepassant la résolution 1973, ont contribué à précipiter le chaos à partir de 2011. Mais depuis, la guerre civile féroce demeure une affaire essentiellement interne à une société tribale qui n'a jamais opéré une véritable intégration nationale. En revanche, les guerres civiles au MoyenOrient, au Yémen et en Syrie surtout, continuent d'être fortement entretenues par le jeu toxique de puissances régionales (Turquie, Arabie saoudite, Iran) et de la Russie. La rivalité de puissance entre Ryad et Téhéran, trop souvent réduite à la fracture entre sunnites et chiites, atteint un paroxysme dans les arènes de Syrie, d'Irak et du Yémen, ancienne «Arabie heureuse » (Arabia félix). Pour l'heure, en Mésopotamie, c'est l'Iran qui semble tenir l'avantage en réussissant à maintenir son corridor territorial de Téhéran à la Méditerranée. 
De son côté, après avoir peu usé directement de son hard power militaire dans la région, l'Arabie saoudite domine au Yémen où l'implication de l'Iran n'est pas d'une évidence première. Mais dans l'ensemble, la « rialpoltik » du royaume faite surtout de soft power (les rials financent des investissements économiques et la propagation d'un islam littéraliste pour ne pas dire obscurantiste) et de hard power indirect (fourniture d'armes) semble avoir perdu du terrain; en tout cas en termes de relais de puissance dans le voisinage mésopotamien du Liban à l'Irak et sans que cela ait à voir avec la chute des prix pétroliers.

De l'autre côté du Levant, la Turquie n'a de cesse de contrôler l'irrédentisme kurde dans les arènes en conflits, notamment la Syrie, pour prévenir tout risque d'amputation de son espace. En choisissant pendant un temps de laisser faire Daech en Syrie pour contrecarrer les Kurdes, elle a maintenant récolté le terrorisme des deux sur son territoire tandis que la « démocrature » turque n'en finit pas avec son virage autoritaire. La tentative de coup d'Etat du 15 juillet 2016 contre Erdogan lui a donné le prétexte pour aller plus loin dans la surveillance de la société et la clôture, déjà entamée au préalable, du champ politique.

Quant à la Russie, c'est sur les cendres de la Syrie qu'elle a accéléré son retour dans l'arène internationale. Sa trajectoire traduit mieux qu'aucune autre l'importance des ruptures entre 1991 et aujourd'hui. Disparue des écrans radars de l'histoire en 1991, elle s'est imposée par son unilatéralisme violent en Syrie comme elle l'a fait «indirectement» en Ukraine à partir de 2013. En intervenant directement auprès de Damas à partir de septembre 2015, jusqu'à participer massivement à la tragédie d'Alep, la Russie s'est installée dans les affaires du monde, et ce par-delà la Syrie et les avantages stratégiques qu'elle y trouve.

Pendant ce temps, les Américains ont organisé leur retrait de l'Irak après les folies de l'administration de George W. Bush dont le projet de State Building s'était mué en un projet de State Destroying. Certes, en 2014, les Etats-Unis y ont renvoyé quelques centaines de soldats pour lutter contre Daech. Mais ces effectifs n'ont rien à voir avec les 130000 que comptait le pays jusqu'en décembre 2011. Si ce retrait du Moyen- Orient s'explique par la volonté d'Obama de se défaire de l'héritage, en forme de fardeau, laissé par son prédécesseur, il découle aussi de la stratégie américaine de pivotement vers l'Asie où les opportunités et les menaces s'y multiplient. Au final, s'il a permis des avancées notoires sur les plans interne et externe, Barack Obama, a laissé un bilan assez maigre au Moyen-Orient, à l'exception de l'important accord avec l'Iran. Sur la Palestine, il n'a pas usé des pressions possibles (mais aurait-il pu sans l'appui du congrès ?) sur une administration israélienne de toute façon très hermétique à toute idée d'inflexion politique. Toutefois, on sait aussi que son secrétaire d'Etat, John Kerry, a exercé 
une médiation active, notamment avec un plan porté dans le plus grand secret en 2016, et qui fut refusé par Netanyahu ${ }^{5}$.

Quant à la Syrie, les Etats-Unis sont restés finalement très en retrait de la tragédie qui s’y déploie. En revanche, le refus d'Obama d'engager des rétorsions en août 2013 contre le régime de Damas qui avait usé de l'arme chimique pourtant fixée comme ligne rouge par Washington a facilité indirectement la montée en puissance de la Russie. Mais cette pusillanimité d'Obama sur la question syrienne n'était-elle pas liée à la trop forte audace d'Hillary Clinton qui avait poussé les Etats-Unis à aller plus loin que la résolution 1973 sur la Libye ?

Que dire enfin de l'Union européenne dont la présence politique au Moyen-Orient et en Afrique du Nord demeure très discrète? Le terme d'effacement conviendrait mieux. L'action humanitaire d'accueil des migrants de Syrie et d'ailleurs semble laissée à ses Etats-membres, l'Allemagne de très loin en tête qui s'est honorée d'une politique d'accueil massive, quand d'autres sont fermés (la France notamment) ou se sont transformés en forteresses xénophobes (pensons à la Hongrie du président Viktor Orban). De son côté, l'action politique de l'Union européenne continue à être sous-traitée par certains pays comme la France dont le rôle est essentiellement déclamatoire après avoir été peu inspirée dans le cas libyen.

Ce renoncement de l'Union européenne vient à un moment de grande fragilité politique et économique pour elle. Une « fatigue d'Europe » s'est installée dans certains Etats et l'un d'eux, la Grande- Bretagne, a décidé de la quitter. Cette crise politique se double d'une crise économique dans les Etats méditerranéens de 1'Europe. Leur trajectoire traduit le passage des promesses au désenchantement qu'on observe dans toute la région depuis 25 ans. Car après avoir bénéficié des dividendes de l'Europe, l'Espagne, l'Italie, le Portugal et la Grèce souffrent considérablement. Notamment parce qu'avec un Euro d'emblée surévalué, leur compétitivité s'est détériorée avec le temps. Sans possibilité de dévaluer, ils n'avaient plus comme moyen pour retrouver de la compétitivité pour leurs exportations que de mettre leurs salaires sous pression. Certes les taux d'intérêt bas permis par la crédibilité de l'Euro permettaient à leurs habitants de s'endetter mais au prix de quoi ? Il en va de même pour ces Etats pour lesquels la crise de 2008 a été fatale. Plus personne ne doute du fait que pour éviter un décrochage à ces Etats méditerranéen, il aurait fallu que l'Union européenne fût plus audacieuse pour accompagner la monnaie unique. En particulier en accompagnant cette Union monétaire par une Union budgétaire qui aurait permis de soutenir les Etats en difficulté, comme cela se

\footnotetext{
${ }^{5}$ Barak Ravid, Kerry Offered Netanyahu Regional Peace Plan in Secret 2016 Summit With al-Sissi, King Abdullah, Haaretz,
} 19 février 2017. 
pratique dans tout Etat fédéral. Certes l'UE n'est pas fédérale, mais la fédération d'Etats-nations chère à Jacques Delors aurait pu l'envisager. Mais ce n'est pas avec une commission dirigée pendant dix ans par Manuel Barroso que ce pas «fédéral » a pu se faire. C'est sans doute moins l'idée d'Europe qui sombre aujourd'hui mais plutôt le modèle bancal adopté qui la conduit vers des rives dangereuses.

\section{Lueurs ?}

Que reste-t-il d'espoirs après ce rapide tour d'horizon d'une période qui s'illustre surtout par ses reculs. Dans l'obscurité qui se dessine, quelques lueurs apparaissent çà et là. Au risque d'une répétition, c'est d'abord l'accord conclu le 14 juillet 2015 avec l'Iran qui permet de remettre cette puissance dans le concert régional. Or quoi qu'en pensent les pouvoirs saoudien et israélien, voire aujourd'hui Donald Trump, l'inclusion de ce pays vaut mieux que son bannissement pour l'équilibre de la région. Mais à quelques mois des présidentielles le président Rohani se trouve actuellement sur la corde raide. Les mesures prises par la nouvelle administration américaine contre les ressortissants iraniens donnent, en effet, de la force aux secteurs iraniens conservateurs qui ne voulaient pas de l'accord sur le nucléaire et, au-delà, d'une normalisation avec le pays qu'ils considèrent encore comme le « grand Satan ».

Dans le monde arabe, si le printemps révolutionnaire a bien déçu, la braise démocratique ne s'est pas éteinte pour autant. Malgré les attentats et la faiblesse des investissements qui entravent l'économie, la Tunisie se présente comme une lueur d'espoir pour ceux qui aimeraient attiser de nouveau cette braise dans leurs foyers nationaux. Des peuples, et parmi eux beaucoup de jeunes et de femmes, ont exprimé un appétit de changement qui n'a pas disparu totalement des arènes politiques arabes et qui est sans doute plus résilient qu'on ne peut parfois le penser. Plus localement, quelques situations semblent offrir quelques perspectives meilleures. $\mathrm{Au}$ Liban, Michel Aoun, quoi qu'on pense des conditions de son élection, a pu enfin combler le 31 octobre 2016 le vide présidentiel qui a duré deux ans et demi. Soutenu par l'Iran, le nouveau président a fait en janvier 2017 sa première visite à Ryad, grande rivale de Téhéran, voulant ainsi renouer avec la vocation libanaise du «pays message ». En Méditerranée orientale, le dialogue inter-chypriote s'est fortement enclenché en vue d'un règlement de paix. Toutefois, l'occupation turque depuis 1974 et la vie séparée pendant 43 ans ne facilitent pas un accord de réunification. Mais la volonté des deux négociateurs (Nicos Anastassiades et Mustafa Akinci) n'est pas à remettre en question. 
Conflit plus ancien, bien plus meurtrier et central dans les relations internationales, le conflit israélo-palestinien s'enkyste certes au fur et à mesure que le temps passe : après la mort du processus de paix au début de la décennie 2000, les opérations israéliennes contre le Hamas dans la Bande de Gaza (Plomb durci en 2008-2009, Pilier de défense en 2012 et Bordure protectrice en 2014), le blocus invivable qui lui est imposé et les faits toujours plus accomplis en Cisjordanie (colonisation, mur de séparation et d'annexion, captation des ressources, entraves économiques) nourrissent le désenchantement. D’un autre côté, le 23 décembre 2016, une résolution du Conseil de sécurité (la résolution 2334), qui dénonce clairement les agissements israéliens et la colonisation en particulier ${ }^{6}$, a pu passer avec la complicité tacite d'une administration Obama fatiguée de la dérive jusqu'au-boutiste israélienne. De même, la présence de 75 Etats à la conférence de Paris le 15 janvier 2017 pour redire que la solution de deux Etats demeure sur la table renvoie Benyamin Netanyahou devant ses responsabilités. Comme à son habitude, il a usé de son habituel ton comminatoire et outrancier pour dénoncer cette conférence. Ce dédain était d'autant plus décuplé que l'arrivée de Donald Trump était imminente.

L'élection du président américain n'est pas une bonne nouvelle pour le multilatéralisme et sans doute pour l'apaisement en Méditerranée, ne serait-ce qu'avec les positions qu'il a prises sur la Palestine. Ne plus vouloir faire de la solution de deux Etats un principe comme vient de le déclarer Donald Trump rompt avec le droit international, ce que le nouveau secrétaire-général de l'ONU, plus audacieux que son prédécesseur coréen, lui a fermement renvoyé.

Cette arrivée du populiste américain s'ajoute à un aréopage international déjà très inquiétant. A défaut d'être une bonne nouvelle, elle pourrait en revanche sonner l'heure d'un sursaut européen qui ne s'est que trop fait attendre. Par delà le ravalement de son principal pilier économique qui vacille sous les coups de boutoirs du néolibéralisme, il est plus que nécessaire pour elle de s'engager vers plus d'intégration politique et sociale; et cela, sans perdre de vue le nécessaire approfondissement démocratique, seul à même de rendre recevable son projet qui demeure à rebours des replis nationaux qui se dessinent. Dans le contexte régional qui nous préoccupe, l'Union européenne se doit aussi de porter une réelle ambition régionale en

\footnotetext{
${ }^{6}$ « Rappelant notamment que l'acquisition de terrritoire par la force est inadmissible(...) Réaffirmant qu'Israël, Puissance occupante, est tenue de respecter les obligations de la IVème Convention de Genève (...) Exige de nouveau qu'Israël arrête immédiatement et complètement ses activités de peuplement dans le territoire palestinien occupé y compris Jérusalem Est (...) pour préserver la solution à deux Etats (...) Demande à tous les Etats de faire une distinction, dans leurs échanges, entre le territoire de l'Etat d'Israël et les territoires occupés depuis 1967 ».
} 
Méditerranée et au Proche-Orient. Mais le calendrier politique des futures élections et la fragilisation de l'idée européenne dans des sociétés à forte "fatigue démocratique ${ }^{7}$ et travaillées par les populismes xénophobes demeurent une lourde hypothèque.

«Quand croît le péril, crô̂t aussi ce qui sauve » écrivait magnifiquement Friedrich Holderlin. Si les périls sont bien là, avec toute leur brutalité, il reste à espérer que les éléments du sursaut, sans doute moins visibles et moins bruyants, sont prêts à se conjuguer.

${ }^{7}$ Raffaele Simone, Si la démocratie fait faillite, Gallimard, 2017. 